\title{
A REVIEW OF DIGITAL WATERMARKING AND COPYRIGHT CONTROL TECHNOLOGY FOR CULTURAL RELICS
}

\author{
Haoyu Liu ${ }^{1}$, Miaole $\mathrm{Hou}^{123 *}$, Yungang $\mathrm{Hu}^{123 *}$ \\ 1 School of Geomatics and Urban Spatial Informatics, Beijing University of Civil Engineering and Architecture, No.15Yongyuan \\ Road, Daxing District, Beijing, 102616-(liuhaoyu, houmiaole, hyg)@bucea.edu.cn \\ 2 Beijing Key Laboratory For Architectural Heritage Fine Reconstruction \& Health Monitoring, No.15Yongyuan Road, Daxing \\ District, Beijing, 102616-915172702@qq.com \\ 3 Engineering Research Center of Representative Building and Architectural Heritage Database, Ministry of Education, \\ No.15Yongyuan Road, Daxing District, Beijing, 102616
}

\section{Commission III, WG III/5}

KEY WORDS: Three-dimensional Model, Copyright Protection, Digital Watermarking, Information Security, Protection of Cultural Relics

\begin{abstract}
:
With the rapid growth of the application and sharing of the 3-d model data in the protection of cultural relics, the problem of Shared security and copyright control of the three-dimensional model of cultural relics is becoming increasingly prominent. Followed by a digital watermarking copyright control has become the frontier technology of 3-d model security protection of cultural relics and effective means, related technology research and application in recent years also got further development. 3-d model based on cultural relics digital watermarking and copyright control technology, introduces the research background and demand, its unique characteristics were described, and its development and application of the algorithm are discussed, and the prospects of the future development trend and some problems and the solution.
\end{abstract}

\section{INTRODUCTION}

3-d laser scanning technology can obtain the point cloud data of object or real scene, and then quickly reconstruct the 3-d model. The development of multimedia and network technology makes 3 -d data model widely used in virtual reality, medical image, industrial design and cultural heritage protection.

In recent years, the problem of cultural relic damage has become more serious. On the one hand, the speed of cultural relics protection has been increased slowly and the number of cultural relics to be protected is huge. On the other hand, unprotected cultural relics are damaged faster and less effective. The three-dimensional data model has been playing an increasingly important role in the protection of cultural relics since it was proposed. However, the data security and copyright protection of the 3-d model are important issues to restrict the further development and application of 3-d models in the protection of cultural relics (Ohbuchi, R., 1998). As a new method to protect multimedia data copyright, digital watermarking technology has received constant attention. It can be embedded into the product by the relevant evidences of the works and the author, and it has some hidden nature, so as to achieve the effect of not affecting the data usage and protecting the author's original benefit.

Therefore, it is necessary and efficient to use digital watermarking as the data security and copyright protection measures for the three-dimensional model of cultural relics. In the study of 3-d model of digital watermarking technology, according to the digital watermarking is visible watermarking can be divided into visible watermarking and invisible watermark, according to the digital watermark attack resistance ability strong and the weak can be divided into the robust watermark and fragile watermark, according to the extracted when do you need the original image can be divided into private watermarking and blind watermarking, the watermark according to the feature set can be divided into the airspace and frequency domain watermarking, as shown in figure 1.This paper summarizes and discusses the current research status and application of digital watermarking technology in 3-d model.

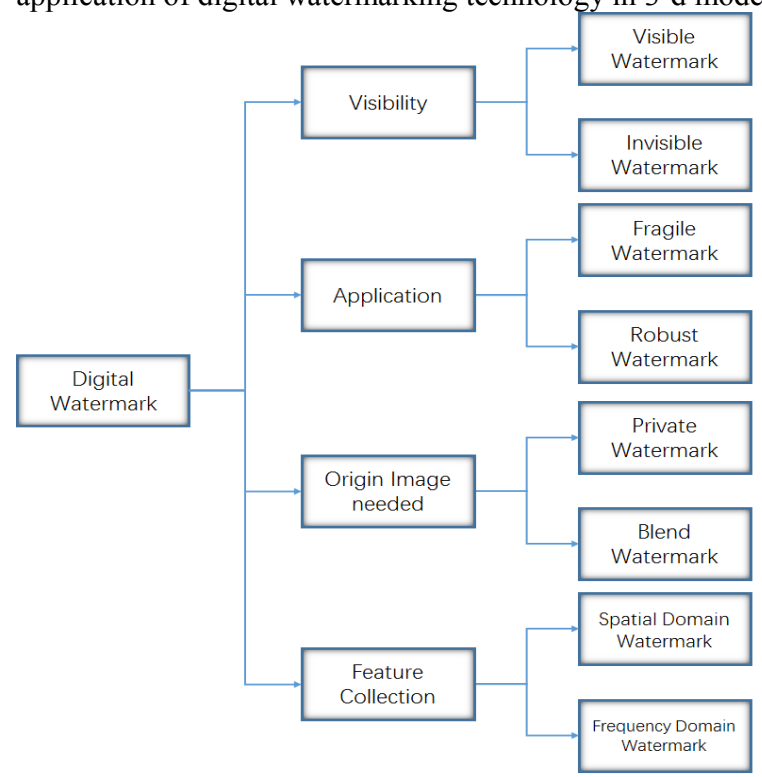

Figure 1.Classification of digital watermark

\footnotetext{
* Corresponding author. E-mail addresses: houmiaole@bucea.edu.cn(Miaole Hou), hyg@bucea.edu.cn(Yungang Hu).
} 


\subsection{Watermark Application}

With the development of digital watermarking in recent years, the scope of its own application has also been expanded(Zhang $X, 2003)$

1.1.1 Copyright protection: For different products, digital watermarking technology can adapt to the characteristics of the product, so as to achieve the copyright protection of the author. Digital watermarking technology has been widely used in video, audio, 2d image, etc. But there is still lack of 3-d model, which needs further research.

1.1.2 Operation tracking: Since the data is relatively easy to copy, in the case of the copyright issues can through the operation of the watermark tracing in tracking the root cause of this problem, ensure the creators and users of copyright issues.

1.1.3 Content authentication: In the process of data use, hard to avoid to produce the problem such as data destruction, damage, so for the contents of the data completeness and correctness of certification is necessary, is advantageous to the copyright protection of the creator.

1.1.4 Data control: In the process of data copy can add watermark to count, to control the count by limiting the use of data to achieve copyright protection, data using the purpose of control and prevent the bulk copy data.

\section{DIGITAL WATERMARKING OPERATION PROCESS}

3-d model of digital watermarking technology is a branch of digital watermarking technology, its principle is embedded in the 3-d model is not the ownership of the visible watermark to protect model, or used for testing the authenticity of the model, or embedded visible information to claim ownership model

3-d model watermarking process can be divided into three steps: watermark generating, watermark embedding and watermark detection, in these three steps, the digital watermarking algorithm research of watermarking embedding and detecting method. From the point cloud model data point of view, watermark embedding can be understood to add a small amount of point cloud data to a point cloud model data, but it will not affect the visualization effect of the point cloud model.

\subsection{Watermark generation}

Watermark can be meaningless data, such as the use of keys by generate the random number sequence of pseudo random generator, or meaningful data, such as the copyright owner's information, user information, etc. Before the watermark is embedded, the watermark data is usually converted into a binary sequence. If you want to further strengthen the security of the watermark data, we can also after binarization of the randomized or encrypt the watermark sequence, so that once appear extracted watermark data by attackers, because we on the watermark data protection, so as to make the attacker can know the content of the watermark data.

\subsection{Watermark embedding}

Watermark embedding is to embed watermark data into threedimensional model by embedding some algorithm into 3-d model, which is shown in figure 2. This process can be controlled by the key to prevent the illegal extraction of watermark, so as to avoid the resumption and modification of watermark by unauthorized users. The selection of watermark embedded base element directly determines the performance of watermarking algorithm. In the various information of 3-d model, geometric information and topological information are suitable for watermark embedding. At present, multi-resolution analysis and other technologies are applied, and the spectral information of the 3-d model is also used for watermark embedding.

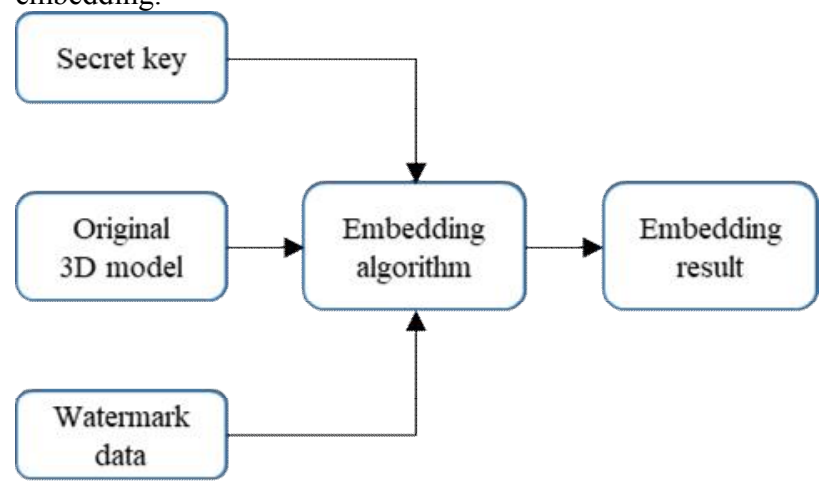

Figure 2.Watermark embedding process

\subsection{Watermark detection}

Watermark detection is the process of extracting watermark information and judging whether it is the original watermark information by using watermark extraction algorithm from the 3-d model to be tested, as shown in figure 3. In this process, the original 3-d model must depend on different watermarking algorithms. The watermark extraction algorithm is often compared with the watermark embedding algorithm, and the embedding method is used to find the location of the watermark embedded in the embedding algorithm to extract the watermark. Due to the 3-d model would inevitably suffered processing, intentionally or unintentionally, thus the integrity of the watermark data is likely affected by different degree, so we must put the extracted watermark data correlation compared with the original watermark data to determine the existence of the original watermark data.

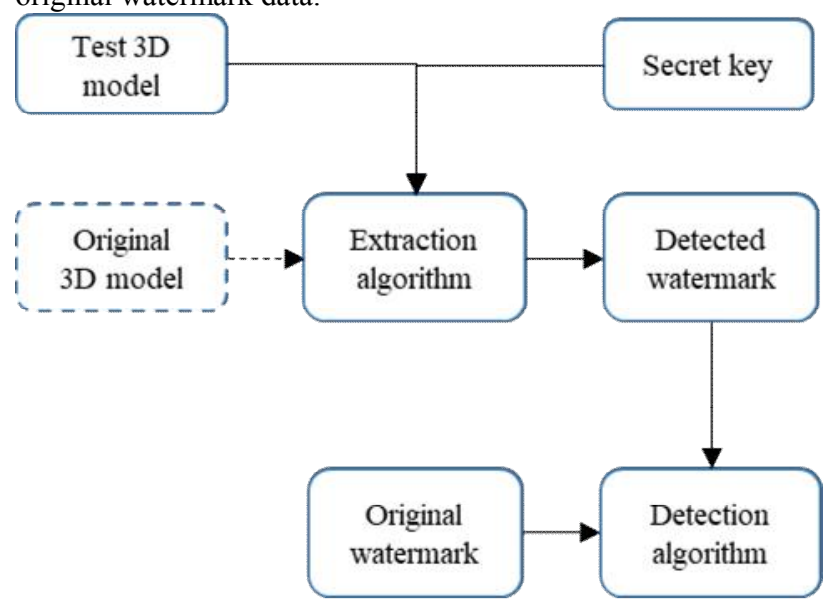

Figure 3.Watermark detection process

\section{THE CHARACTERISTICS OF DIGITAL WATERMARKING OF THE THREE-DIMENSIONAL MODEL OF CULTURAL RELICS}

Digital watermarking general process including the watermark embedding and watermark extraction of two parts, the 
watermark embedding part, need to use watermark information and need to embed watermark to manipulate data source, to embed the watermarking information in the source data. The watermark extraction process is used after embedding watermark data is extracted, and if the blind watermarking algorithm, do not need to source data were extracted, if is a blind watermark extraction processes require the participation of the source data can be extracted successfully, the basic flow diagram as shown in the figure below.

Compared with text and image, the 3-d model has remarkable characteristics, such as large amount of data and complicated structure. In addition, the accurate geographic information is the basic guarantee for the wide application of 3-d model data. Therefore, 3-d model of digital watermarking technology should satisfy not only standard text, image digital watermark basic technical requirements (such as robustness, embedding validity, uniqueness, data capacity, etc.), in principle should also meet the requirements of the following aspects(Huang X., 2003).

\subsection{Precision characteristics}

The data of three-dimensional point cloud model is the most accurate three-dimensional data of the entity. In fact, in the protection of cultural relics and architectural heritage. Cultural relics are the treasures of human civilization, containing the unique spiritual values, thinking patterns and imagination of an era, which are the crystallization of human civilization, culture and wisdom. However, with the passage of time, most of the relics are incomplete, broken, incomplete and so on.

The high-precision original point cloud data is the real virtual representation of cultural relics, and it is often the premise of the follow-up collaborative research in addition to the basic 3-d information storage. The detection and labeling of diseases caused by small changes of cloud at different time points; In the virtual 3-d model, the project formulation, adjustment, implementation and evaluation of results are completed, and the virtual restoration of test materials and reference bases is provided for the actual operation. Therefore, ensure the accuracy of cultural relics of the 3-d model data is in the watermark embedding problem must be considered when, and does not affect the use of its late (especially disease detection, virtual repair, deformation, etc..

\subsection{High efficiency}

The data volume of 3-d model is generally relatively large, and the order of magnitude is usually several GB to dozens of GB. Therefore, compared with conventional digital watermarking, the digital watermarking scheme of 3-d model should be more efficient and used to meet the practical application requirements.

\subsection{Detectable}

Digital watermarks should be able to be detected or extracted by authors or other notaries. When the work is in dispute on the copyright issue, the copyright of the work can be confirmed by extracting the watermark information in the works. If watermark is not detectable, then it loses its original meaning.

\subsection{Without Ambiguity}

The result of a watermark or watermark decision should be able to indicate the ownership of the ownership and not to detect inaccurate information.

\subsection{Imperceptibility}

This refers to the significant decrease in the information quality of the protected information and the visual effects after embedding the watermark information. For invisible watermarks, this is a basic requirement. And the robustness and imperceptibility are two contradictory features that interact and constrain each other. But because of people's perception system is not very accurate, the so-called imperceptible in fact is relative, not absolute cannot be perceived, as long as it is for people to notice the obvious change can be called is imperceptible. The current technique is usually to improve the robustness of watermarks as much as possible under the premise of "relative" insensitivity(Li Y. C., 2011).

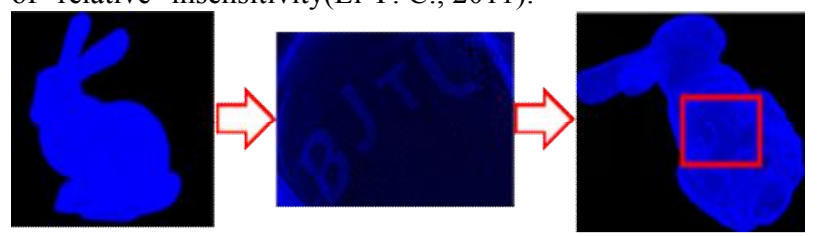

Figure 4.Watermark Imperceptibility

However, the technical requirements of 3-d model digital watermarking are often mutually influenced and even contradictory. For example, the smaller the data volume, the worse the robustness. The larger the data volume, the weaker the fidelity, and the accuracy will be affected. In practice, therefore, to achieve the above technical requirements, is designed for 3-d model data characteristics of digital watermarking scheme, between the various technical indicators according to the characteristics of the current specific goals and requirements to comprehensively consider the effect of between each technology, different watermark usually comes with its own characteristics. For example, the fragile watermark robustness requirement is not high, and the robustness of watermark in addition to meet the requirements of the above, but also satisfy the robustness and can resist common attack multiple, to ensure that the embedded watermark can be extracted correctly.

\section{THE RESEARCH STATUS}

At present, the research progress in the airspace and frequency domain digital watermarking is more, the method based on spatial domain is by modifying the model geometry information, topology information and other properties to embed watermark, the watermark embedding and extraction speed, methods mainly include the method based on model vertex, based on polygon area such as the method of geometric elements and the method based on two-dimensional watermark extension. However, the method of spatial domain is to operate directly on the geometric invariant of the model, which has a large influence on the invisibility of watermark information, and the spatial watermark is generally sensitive to noise.

Based on the frequency domain method, the model is firstly transformed, and the frequency domain coefficients obtained are modified to embed watermark. Based on frequency domain method is mainly differences on mathematical transformation, such as based on three dimensional discrete cosine transform (3-d - DCT) and DCT coefficient of bipolar quantitative technology put forward a kind of three-dimensional data model of embedding method, the method to realize a blind extraction, able to deal with the common attacks such as cut, add noise, but unable to cope with big strength of geometric attack. Or by combining quantitative modulation strategy and dual tree complex wavelet transform, embedding the watermark into the 
key entropy image area, enhances the watermark's ability to cope with compression attack, but the ability to resist geometric attacks. The frequency domain method is used to operate the frequency domain coefficients. Compared with the watermark of the airspace, the frequency domain watermark has the characteristics of resisting all kinds of attack means, good robustness, high fidelity, and larger watermark information capacity.

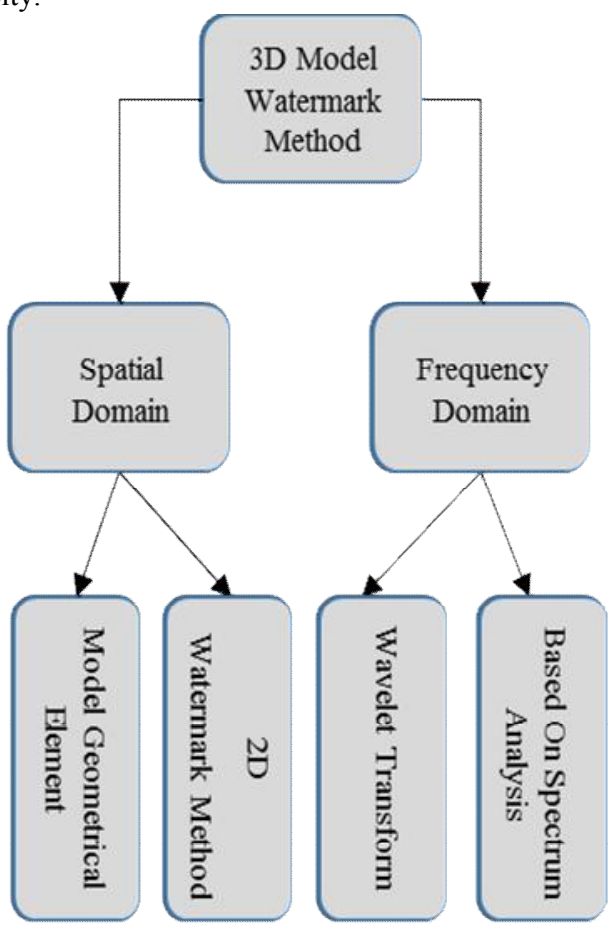

Figure 5.3-d watermarking method

Both wavelet transform and other mathematical transformation, mostly on the certain properties of the model change to embed watermark, and directly to model the triangle mesh in triangular domain orthogonal system on the mathematical transformation is obviously much more convenient. Using V - system parameterization of triangular mesh model in the frequency domain spectrum, by adjusting the frequency spectrum to embed the watermark, the algorithm of similarity transformation, such as noise, shear attack has better robustness. Or will be introduced to the combination of Laplacian three-dimensional grid algorithm, by using the method of Laplace basis function mathematical transform, this method only depends on the grid topology information, and has nothing to do with the geometry information, thus the watermarking grid, especially not rules grid watermark visual quality is affected by a large grid. In 2002 Adrian et al. proposed a blind watermarking algorithm for 3-d models and objects. It generates a string by the key, which is embedded in the geometry of the object by changing the location of some vertices. This algorithm has good invisibility.

Cotting etc. (Cotting D., 2004) in 2004 put forward a point sampling geometry model based on spectrum analysis of robust watermarking scheme, USES the fast hierarchical clustering algorithm, the point cloud model is first divided into a series of small, and mapped to an approximation of the Laplacian characteristic function space, the final selection of spectrum of low frequency part of watermark embedding, the algorithm for smoothing, such as rotation, translation, scaling attack has better robustness.
2004, Ohbuchi, etc. (Ohbuchi R., 2004)to (Karni, Z., 2000) proposed a grid based on spectrum analysis technology, converting point model into grid model, using the correlation between vertex with spectrum analysis to grid, ultimately embed watermark data. But strictly speaking, this algorithm is not really a direct digital watermarking algorithm for point cloud model, the algorithm for cutting, noise, resampling, etc have good robustness, but in view of the similarity transformation attack, if a model for the symmetry model leads to poor robustness against the attack.

In 2011, Wang Xinyu etc. (Wang Xinyu, 2011) change to weaken the 3-d model data at the expense of precision, the 3-d model data itself routine minor adjustments to embed watermark information, this paper proposes a 3-d point cloud model zero watermarking scheme. Combined with the characteristics of three-dimensional point cloud model, in order to have higher the stability of the global geometric features 3-d point cloud model as the breakthrough point, through to the number of vertex concentration in accordance with certain conditions of analysis to construct the watermark. The watermark information is registered in the information database of Intellectual Property Right, a third-party authority, and finally the copyright protection of 3-d point cloud model data is realized. The algorithm has good invisibility and good robustness to common watermark attacks such as translation, rotation and noise, but with the increase of shear attack strength, the robustness decreases.

In 2013 Wang rui, etc.(Wang rui, 2017) breakthrough is dependent on a specific coordinate system for the limitation of watermarking scheme design, the introduction of Clifford geometry kangaroo, put forward a kind of three-dimensional point cloud based on Clifford - Fourier transform model of digital watermarking algorithm. The data watermark of 3-d point cloud model is calculated without dependence on specific coordinate system. It has better non - visible features, and has good robustness for common attacks such as translation, rotation, simplification, etc.

In 2017, Shiqun Li etc. (Shiqun Li, 2017) proposes a gridbased Laplacian characteristic vector of a three-dimensional grid model and a half blind watermarking algorithm, the watermark embedding phase, Tutte Laplacian calculation, then the eigenvalue decomposition characteristic vector is obtained, the disturbance of Laplacian matrix eigenvector for watermark embedding. This algorithm can resist the common attacks such as affine transformation, random noise, smoothing, uniform quantization, cutting and so on, which has strong robustness and greatly improves the watermark load capacity.

In 2018 the GN Pham (GN Pham, 2018) proposes a watermark embedding algorithm based on the three dimensional model of feature points, through the 3-d print model of $Z$ axis 3-d section for calculation and determination process of feature points, according to the reference length changed foxy feature points in the space vector length to embed the watermark data model for 3-d printing a feature point. The $\mathrm{x}$ and $\mathrm{y}$ coordinates of the feature points will change depending on the length of the change vector that has been embedded in the watermark. This algorithm has good robustness and invisibility for rotation, scaling, translation and random noise, and the algorithm has good precision.

\section{SUMMARY AND PROSPECT}

At present, the 3-d model of digital watermarking has achieved great results, but the technology is not fully mature, and there are still many problems to be solved and perfected. 
First, in terms of theory, and the world is still in the early, intermediate stage, the theory still has a lot of to break through the key problems, such as robustness, embedding strength, embedding the relationship between the data volume and not perceptual. How to coordinate the influence of various aspects, improve the overall effect of watermark is more important.

Second, the attack on $3-\mathrm{d}$ model is varied, due to the need of copyright protection, the watermark needs has good robustness to deal with all kinds of attacks in place to ensure that the embedded watermark information will not be destroyed, to ensure the legitimate rights and interests of the author.

Thirdly, the research on digital watermark of 3-d model is more than that of grid model, and the research on digital watermark technology of point cloud model is relatively small. In addition, the study of blind watermarking algorithm is relatively weak, although the robustness of the non-blind watermarking algorithm is better, but the effect is poor in the actual application, and the robustness of the blind watermarking algorithm is also needed.

Fourthly, the application of deep learning in the digital watermarking of cultural relic 3-d models is still just starting. If it can build a better model of robustness, it will produce good results. At present, Stanford university first proposed the use of deep learning to carry out the generation of digital watermarking of point cloud model, and achieved good results.

\section{CONCLUSION}

With the increasingly prominent application value of 3-d model data in the protection of cultural relics and the increasing demand of 3-d model data sharing, the security of the 3-d model is becoming more and more important. As a cutting-edge technology in data security protection, digital watermarking technology plays an important role in the security protection, copyright protection and data sharing of cultural relic 3-d models. Only to guarantee the data security problems, the creator of the copyright benefit is protected, to make the cultural relics of the 3-d model of data sharing and widespread application, in order to promote the development of the protection of cultural relics.

\section{REFERENCES}

Cotting, D., Weyrich, T., Pauly, M., \& Gross, M. (2004). Robust watermarking of point-sampled geometry. Shape Modeling Applications, 2004. Proceedings (pp.233-242). IEEE.

Huang, X., \& Shi, L. (2003). Robust blind watermarking algorithm for 3-d motion. Computer Engineering.

Karni, Z., \& Gotsman, C. (2000). Spectral compression of mesh geometry. (Vol.180, pp.279-286). ACM SIGGRAPH.

Li, Y. C., \& Zhao, L. F. (2011). Digital watermarking technology in information security. Advanced Materials Research, 282-283, 55-58.

Ohbuchi, R., Mukaiyama, A., \& Takahashi, S. (2004). Watermarking a 3-d Shape Model Defined as a Point Set. International Conference on Cyberworlds (pp.392-399). IEEE Computer Society.

Ohbuchi, R., Masuda, H., \& Aono, M. (1998). Watermarking three-dimensional polygonal models through geometric and topological modifications. Selected Areas in Communications IEEE Journal on, 16(4), 551-560.
Pham, G. N., Lee, S. H., Kwon, O. H., \& Kwon, K. R. (2018). A 3-d printing model watermarking algorithm based on 3-d slicing and feature points. , 7(2), 23.

Rui, W., Jin, L. B., Lei, T., Guo, S. L., \& Wan, W. G. (2013). Digital watermarking algorithm for 3-d point cloud model based on clifford algebra. Journal of Shanghai Jiaotong University, 47(12), 1863-1869.

Shiqun, L. I., Guiqing, L. I., \& Xian, C. (2017). Eigenvectorbased watermarking for 3-d mesh models. Journal of Graphics.

Wang, X., \& Zhan, Y. (2011). Robust zero watermarking scheme for 3-d point model. Computer Engineering \& Applications, 47(28), 7-11.

Zhang, X. (2003). Review of watermarking techniques for 3-d polygonal models. Journal of Computer Aided Design \& Computer Graphics. 